\title{
Optimized saturation recovery protocols for T1-mapping in the heart: influence of sampling strategies on precision
}

\author{
Peter Kellman ${ }^{1 *}$, Hui Xue ${ }^{1}$, Kelvin Chow ${ }^{3}$, Bruce S Spottiswoode ${ }^{2}$, Andrew E Arai ${ }^{1}$ and Richard B Thompson ${ }^{3}$
}

\begin{abstract}
Background: T1-mapping has the potential to detect and quantify diffuse processes such as interstitial fibrosis. Detection of disease at an early stage by measurement of subtle changes requires a high degree of reproducibility. Initial implementation of saturation recovery (SR) T1-mapping employed 3-parameter fitting which was highly accurate but was quite sensitive to noise; 2-parameter fitting greatly reduced the sensitivity to noise at the expense of a small degree of systematic bias. A recently introduced implementation that uses a variable readout flip angle greatly reduces systematic errors in T1-measurement thereby making it feasible to use SR methods with 2-parameter fitting with improved accuracy and precision. SR T1 mapping techniques with multi-heartbeat recovery times have been proposed to better sample the $\mathrm{T1}$ recovery curve, but have not been evaluated for 2-parameter fitting.

Methods: An analytic formulation for calculating the standard deviation (SD) for SR T1-mapping with 2-parameter fitting is developed and validated using Monte-Carlo simulation. The coefficient of variation is compared for a brute force optimization of sampling and for several previously described sampling schemes for T1 measurement over several uncertainty ranges. Experimental validation is performed in phantoms over a range of $\mathrm{T} 1$, and in-vivo both native and post-contrast. Pixel-wise SD maps are calculated for SR T1-mapping.
\end{abstract}

Results: Sampling schemes that use a non-saturated anchor image and multiple (N) measurements at a single fixed saturation delay are found to be near optimum for the case of known $\mathrm{T} 1$ and are close to the brute force optimized solution over wide ranges of native and post-contrast $\mathrm{T} 1$ values. The fixed delay sampling scheme is simple to implement and provides an improvement over uniformly distributed schemes.

Conclusions: Sampling strategies for saturation recovery methods for myocardial T1-mapping have been optimized and validated experimentally. Reduced SD, or improved precision, may be achieved by using fixed saturation delays when considering native myocardium and post-contrast T1 ranges. Pixel-wise estimates of T1 mapping errors have been formulated and validated for SR fitting methods.

Keywords: T1 mapping, Random error, Precision, SASHA, Saturation recovery, Myocardial fibrosis

\section{Background}

T1-mapping has the potential to detect and quantify diffuse myocardial processes such as interstitial fibrosis. Detection of disease at an early stage by measurement of subtle changes requires a high degree of reproducibility [1]. Reproducibility is fundamentally limited by precision and may be further limited by systematic variations

\footnotetext{
*Correspondence: kellman@nih.gov

1 National Heart, Lung, and Blood Institute, National Institutes of Health, DHHS, 10 Center Drive MSC-1061, Bethesda, MD 20892, USA

Full list of author information is available at the end of the article
}

$[2,3]$. The recently proposed SAturation recovery with single-SHot Acquisition (SASHA) method [4] has been introduced in an effort to reduce systematic measurement biases [2,3] due to factors such as off-resonance and flip angle variation, dependence on $\mathrm{T} 2$, heart rate, and sensitivity to protocol parameters compared to the MOdified Look-Locker Inversion recovery (MOLLI) sequence [5]. SASHA has also been shown to be less dependent on magnetization transfer (MT) than MOLLI and its variants [6]. Initial implementation of SASHA T1-mapping employed 3-parameter fitting which was highly accurate 
but was quite sensitive to noise; 2 -parameter fitting greatly reduced the sensitivity to noise at the expense of a small degree of systematic bias $[3,4]$. The bias error arises due to the influence of the readout pulses following the saturation pulse that precede the center of k-space. A recently introduced implementation of SASHA [7] that uses a variable readout flip angle (VFA) greatly reduces systematic errors in T1-measurement thereby making it feasible to use 2-parameter fitting with improved accuracy and precision. The use of VFA reduces the influence of the readout prior to reaching the center of k-space since the flip angle is reduced during this period. VFA also reduces image artifacts that arise due to oscillations during the transient approach to steady state, particularly the ghosting of fat due to offresonance.

SASHA was originally described with saturation recovery times (TS) limited to a single heartbeat to allow the maximum number of images to be acquired within a given duration. Longer TS times can be obtained by playing the image readout in the heartbeat following the saturation pulse, as previously described for the SR-TFL sequence [8] and more recently in Saturation Method using Adaptive Recovery times for cardiac T1 Mapping (SMART1Map) $[9,10]$. These multi-heartbeat TS times have the advantage of sampling a greater portion of the recovery curve, although fewer images are acquired for a fixed total duration. The net effect in precision of calculated $\mathrm{T} 1$ values has not been previously explored.

Precision relates to random errors due to noise and is a function of the number and timing of measurements along the T1-saturation recovery curve. We examine the effect of sampling on precision including the position (i.e., SR delay TS) and number of samples, and propose an optimized sampling scheme to reduce the error due to noise. We provide an analytic formulation for the calculation of the standard deviation (SD) for 2-parameter saturation recovery fitting and validate this formulation using Monte-Carlo simulation. Pixel-wise maps of noise SD can be used as a quality or confidence map and may be generated using this formulation in the same manner as for inversion recovery SD maps [11]. A comprehensive analysis and optimization has been described for fitting with 3 parameters $[12,13]$ that leads to different results, however 3 parameter fitting is not considered here since it is significantly more sensitive to noise which greatly reduces precision [3].

We proposed a sampling strategy that uses a nonsaturated anchor image and $\mathrm{N}$ measurement at a fixed saturation delay (TS). We calculate the optimum fixed TS for a given T1 and number of measurements $\mathrm{N}$, and correspondingly determine the optimum achievable
SD. We compare sampling strategies where $\mathrm{T} 1$ is in a known range and consider a range for native myocardial T1 mapping, a range for post-contrast, and a wider range spanning T1 values for both native and post-contrast. Sampling strategies that were compared include using a non-saturated anchor image plus a) fixed TS for all measurements, b) uniform distribution of TS over the heart interval [4], c) brute force optimization, and d) strategies employing multiple recovery heartbeats to obtain longer TS samples [10].

Comparisons of protocols with different sampling strategies are validated in phantoms and in-vivo. Experimental measurements are in excellent agreement with numerical prediction based on theory.

\section{Methods}

\section{Theory}

The 2-parameter model for saturation recovery may be written as:

$$
y(\mathrm{TS})=A\left(1-\exp \left(-\frac{T S}{T 1}\right)\right)
$$

where $y$ (TS) is longitudinal magnetization, $\mathrm{T} 1$ is the longitudinal recovery time constant, TS is the saturation recovery time and $\mathrm{A}$ is the signal amplitude. The desired covariance matrix $C$ of the estimated parameters ( $A$ and T1) may be approximated as [11]:

$$
\begin{aligned}
& \mathbf{C}=\left[\begin{array}{cc}
\sigma_{T 1}^{2} & \cdot \\
\cdot & \sigma_{A}^{2}
\end{array}\right] \\
& =\left(\sum_{i} \frac{1}{\sigma_{i}^{2}}\left[\begin{array}{lll}
\frac{\partial y\left(T S_{i}\right)}{\partial T 1} \cdot \frac{\partial y\left(T S_{i}\right)}{\partial T 1} & \frac{\partial y\left(T S_{i}\right)}{\partial T 1} \cdot \frac{\partial y\left(T S_{i}\right)}{\partial A} \\
\frac{\partial y\left(T S_{i}\right)}{\partial A} \cdot \frac{\partial y\left(T S_{i}\right)}{\partial T 1} & \frac{\partial y\left(T S_{i}\right)}{\partial A} \cdot \frac{\partial y\left(T S_{i}\right)}{\partial A}
\end{array}\right]\right)^{-1}
\end{aligned}
$$

where $T S_{i}$ are the saturation delays for each sample $i$, and $\sigma_{i}=\sigma$ is the standard deviation of the signal y which are assumed to be independent and identically distributed; $\sigma_{T 1}^{2}$ and $\sigma_{A}^{2}$ represent the variance of $\mathrm{T} 1$ and $\mathrm{A}$, respectively. The partial derivatives in Eq 2 for the 2-parameter signal model Eq 1 are:

$$
\begin{aligned}
& \frac{\partial y}{\partial A}=1-\exp \left(-\frac{T S}{T 1}\right) \\
& \frac{\partial y}{\partial T 1}=-A \cdot \exp \left(-\frac{T S}{T 1}\right) \cdot \frac{T S}{T 1^{2}}
\end{aligned}
$$

The desired variance for the parameter T1 may be calculated using the analytic $2 \times 2$ inverse of Eq 2 . 


$$
\sigma_{T 1}^{2}=\sigma^{2} \frac{\sum \frac{\partial y\left(T S_{i}\right)}{\partial A} \cdot \frac{\partial y\left(T S_{i}\right)}{\partial A}}{\sum \frac{\partial y\left(T S_{i}\right)}{\partial T 1} \cdot \frac{\partial y\left(T S_{i}\right)}{\partial T 1} \sum \frac{\partial y\left(T S_{i}\right)}{\partial A} \cdot \frac{\partial y\left(T S_{i}\right)}{\partial A}-\left(\sum \frac{\partial y\left(T S_{i}\right)}{\partial T 1} \cdot \frac{\partial y\left(T S_{i}\right)}{\partial A}\right)^{2}}
$$

After substituting the partial derivatives in Eq 3 into Eq 4, assuming there are $\mathrm{N}$ measurements at $T S_{i}$ plus a non-saturated anchor image at $T S=\infty$, and simplifying, one may derive an expression for the square of the coefficient of variation times SNR, $\left(\frac{\sigma_{T 1}}{T 1} \times S N R\right)^{2}$ :

$$
\left(\frac{\sigma_{T 1}}{T 1} \times S N R\right)^{2}=\frac{1}{\left(\sum_{i=1}^{N}\left(\exp \left(-\frac{T S_{i}}{T 1}\right) \cdot \frac{T S_{i}}{T 1}\right)^{2}\right)-\frac{\left(\sum_{i=1}^{N}\left(\exp \left(-\frac{T S_{i}}{T 1}\right) \cdot \frac{T S_{i}}{T 1}\right) \cdot\left(1-\exp \left(-\frac{T S_{i}}{T 1}\right)\right)\right)^{2}}{1+\sum_{i=1}^{N}\left(1-\exp \left(-\frac{T S_{i}}{T 1}\right)\right)^{2}}}
$$

where $\mathrm{SNR}=\mathrm{A} / \sigma$. Thus the coefficient of variation $\frac{\sigma_{T 1}}{T 1}$ is a function of T1, SNR, and the saturation delays $T S_{\mathrm{i}}$ and may be readily evaluated numerically.

\section{Sampling strategies and notation}

In order to compare various sampling strategies for SASHA that includes the possibility for recovery heartbeats to permit saturation delays TS $>R R$ (the duration of a single cardiac cycle) as used in $\mathrm{SMART}_{1}$ Map [10], a notation is introduced here. All of the protocols considered here acquire an initial non-saturated image referred to as NS as well as additional measurements at various saturation delays. For instance, the SASHA protocol described in the original publication [4] would acquire a total of 10 images consisting of NS plus 9 additional SR images would be written here as NS + $[(0) 1]^{9}$ uniform, where the $(0)$ indicates there were no recovery beats between SR image measurements, and the []$^{9}$ indicates 9 measurements distributed uniformly across the RR interval. The SMART ${ }_{1}$ Map acquisition scheme described in [8] can be written as NS $+[(0) 1]^{3}(1) 1(2) 1(3) 1$ uniform, where there is a NS plus 3 images acquired without recovery with uniformly distributed saturation delays followed by 3 additional images acquired with 1, 2, and 3 , recovery beats, respectively, assumed to be acquired at the maximum available saturation delay for the given heart rate. This would correspond to a total acquisition of 7 images in 13 heartbeats including the NS and 6 recovery beats for which there are no images acquired. For comparison of SD of T1-measurements between these different protocols, the total acquisition time was considered fixed. Since the reported $\mathrm{SMART}_{1} \mathrm{Map}$ protocol was 13 heart beats, a 13 heart beat SASHA protocol was used for comparison. Therefore, 13 heartbeat SASHA protocols were considered NS $+[(0) 1]^{12}$ with both uniformly distributed TS as in the original SASHA [4] and a modified protocol with fixed TS, i.e., all measurements at the same saturation recovery delay chosen through numerical optimization to be described. These protocols are illustrated in Figure 1(a-c). Multiple measurements at the same saturation delay were not averaged. Fitting was performed on all measurements including the multiple measurements at the same saturation delay, TS.

\section{Optimization of sampling strategies}

The coefficient of variation (CV) was evaluated per Eq 5 for a number of cases. Firstly, it was assumed that T1 was known and the optimum sampling strategy was determined. Alternatively, it was assumed that T1 was known in a finite range and the optimum sampling strategy was determined over ranges corresponding to native contrast, post-contrast, and a wide range which encompassed both native and post contrast. The ranges were chosen broadly to be $1000-1400 \mathrm{~ms}$ (native), $250-600 \mathrm{~ms}$ (post-contrast), and $250-1400 \mathrm{~ms}$ (wide) to encompass a range of conditions. Optimization consisted of choosing a set of saturation delays, $\mathrm{TS}_{\mathrm{i}}$, and computing the $\mathrm{CV}$ across the specified range of T1. A brute force search was conducted by calculating all possible sets of delays $\mathrm{TS}_{\mathrm{i}}$ selected over a grid from TS min to TS max with replacement, i.e. allowing repeated TS values. The scheme with the minimum worst case CV over the $\mathrm{T} 1$ range was selected as optimal. For example, if one were to specify TS $\mathrm{min}=$ $100 \mathrm{~ms}$ and TS $\max =800 \mathrm{~ms}$, with an increment of $50 \mathrm{~ms}$, then there would be $\mathrm{N}_{\text {incr }}=15$ possible values for TS. For a protocol such as NS $+[(0) 1]^{12}$ with $\mathrm{N}_{\mathrm{TS}}=12$ values chosen over $\mathrm{N}_{\mathrm{incr}}=15$, the number of possible cases with replacement would be:

$$
\begin{aligned}
\text { Ncases } & =\left(\left(\begin{array}{c}
\text { Nincr } \\
N_{T S}
\end{array}\right)\right)=\left(\begin{array}{c}
N_{T S}+\text { Nincr }-1 \\
N_{T S}
\end{array}\right) \\
& =\frac{\left(N_{T S}+\text { Nincr }-1\right) !}{N_{T S} !(\text { Nincr }-1) !}
\end{aligned}
$$

or in this example, 9,657,700. Calculations were performed using MATLAB (Mathworks, Natick, MA USA) and Eq 5 was vectorized to compute the max (CV) across a range of T1's in $10 \mathrm{~ms}$ increments. In this example, the brute force calculation for the native or post-contrast $\mathrm{T} 1$ ranges would test all the cases in approx. $2.5 \mathrm{~min}$, with each case calculated in less than 16 microsec. The brute force optimization was performed at HR $=60 \mathrm{bpm}$ using Nincr $=15$ samples between TS $\min =100$ and TS $\max =800$, and at $H R=120 \mathrm{bpm}$ using Nincr $=15$ samples between TS $\min =100 \mathrm{~ms}$ and TS $\max =350 \mathrm{~ms}$. The brute force optimization was extended to include up to 8 recovery beats. All possible combinations of recovery/acquisition were considered that summed to a total duration of 12 beats. Values of TS for measurements that followed recovery were assumed to be maximized, i.e., equal to Nrecovery"RR + TS max. Brute 
(a)

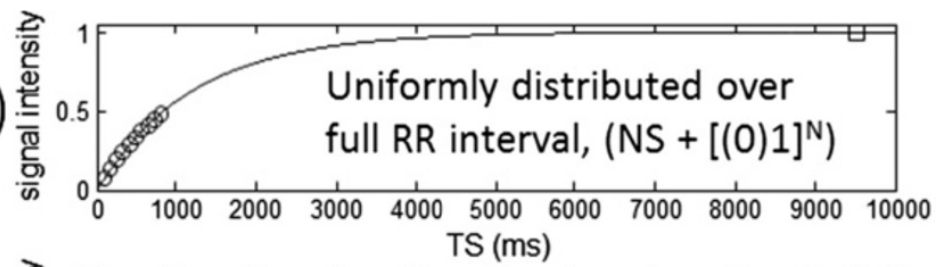

(b)

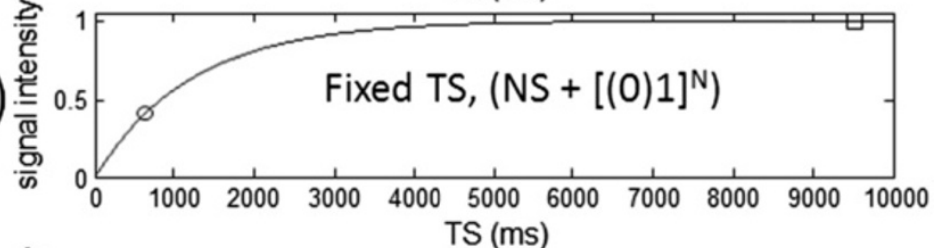

(c)

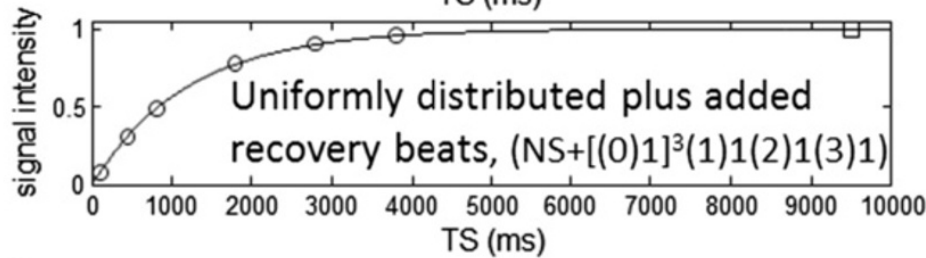

(d)

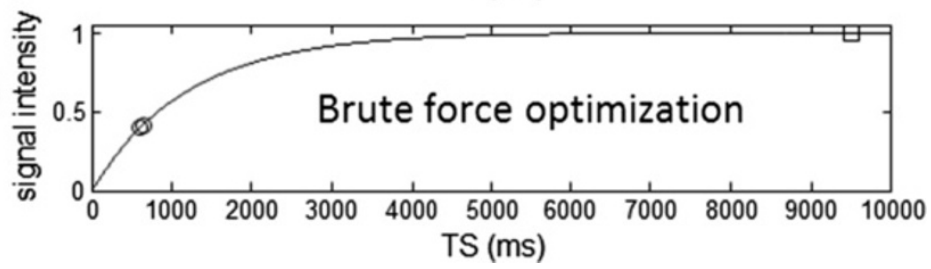

Figure 1 Saturation recovery sampling strategies considered in protocol comparisons: (a) uniformly distributed saturation delays, (b) fixed saturation delay, (c) uniformly distributed saturation delays plus added recovery beats, and (d) brute force optimization of sampling. All schemes acquired an initial non-saturated "anchor" image (plotted at $9500 \mathrm{~ms}$ ) and all protocol comparisons had equal duration acquisitions (RR $=1000 \mathrm{~ms}$ in this illustration).

force optimizations were calculated for HR's 60 and $120 \mathrm{bpm}$. The brute force optimization was performed both considering recovery heartbeats and without any recovery heartbeats.

The sampling strategies considered are illustrated in Figure 1 and panel (a) with uniform distribution over the RR corresponds to the original published SASHA scheme [4].

\section{Numerical validation of SD formulation}

A Monte-Carlo simulation using $\mathrm{N}=65,536$ trials was used to compute the standard deviation (SD) in T1 as a function of SNR and T1 for a specific SASHA protocol $\left(\mathrm{NS}+[(0) 1]^{12}\right)$ and was compared with the estimate of standard deviation based on the analytic formulation.

\section{Imaging}

Imaging was performed on a $1.5 \mathrm{~T}$ Siemens Aera (Siemens Medical Solutions, Erlangen, Germany), equipped with a $45 \mathrm{mT} / \mathrm{m}$ and $200 \mathrm{~T} / \mathrm{m} / \mathrm{s}$ gradient systems. The original uniform SASHA sampling strategy, the fixed TS sampling strategy, and the $\mathrm{SMART}_{1}$ Map sampling strategy with recovery beats were used. For phantom imaging, the fixed TS sampling strategy had 3 protocols: (a) optimized for native (pre-contrast) myocardial T1 values, (b) optimized for shorter T1 corresponding to Gd contrast, and (c) optimized for the wide range of $\mathrm{T} 1$ covering both pre- and post-contast ranges. All protocols had an acquisition of 13 heart beats. All in-vivo imaging was acquired using breath-holding. Non-rigid motion correction was used to correct any residual in-plane respiratory motion.

Imaging parameters for all sampling schemes were: non-selective adiabatic saturation pulse, steady state free precession single shot read out with variable flip angle [7] which smoothly approached a $70^{\circ}$ excitation flip angle, typical field of view $360 \times 270 \mathrm{~mm}^{2}$, slice thickness $8 \mathrm{~mm}$, matrix $256 \times 144$, voxel size $1.4 \times 1.9 \times 8.0 \mathrm{~mm}^{3}$, TR/TE $2.7 / 1.1 \mathrm{~ms}, 7 / 8$ partial Fourier plus parallel imaging factor 2 using separate reference lines acquired at the completion of the scan. The BIR4-90 saturation pulse was designed to achieve saturation to within $0.6 \%$ over an off-resonance range $\pm 125 \mathrm{~Hz}$ and between 75 and $100 \%$ of design flip angle.

\section{Phantom measurements}

Phantom validation used a set of $\mathrm{CuSO} 4$ doped agar gel phantoms with varying concentrations with T1 and T2 in the expected range for myocardium, both native and 
with Gd contrast. Phantoms had T1 in the range 250$1600 \mathrm{~ms}$ and $\mathrm{T} 2$ in the range $40-75 \mathrm{~ms}$. The agar tubes were in a bath of saline doped with Gd with T1 approx. $220 \mathrm{~ms}$.

The T1, SD, and SNR were measured in 5 ROIs with varying $\mathrm{T} 1$ and the measured values of $\mathrm{T} 1$ and SNR were used for numerical calculation of a predicted SD, which was compared with the measured SD in each ROI. The methodology is illustrated in Figure 2. Images were reconstructed with scale in SNR units $[14,15]$. SNR maps were calculated from the signal intensities of the non-saturated image.

\section{In-vivo studies}

In-vivo data was acquired using 3 imaging protocols with the same 3 sampling strategies defined above (original SASHA, fixed TS, and $\mathrm{SMART}_{1} \mathrm{Map}$ ) to compare the T1 map SD for both native myocardial T1 and following administration of contrast. In-vivo imaging used the native contrast optimized protocol for native contrast imaging ( $\mathrm{TS}=600 \mathrm{~ms}$ ) and the post-contrast optimized protocol for post-contrast imaging ( $\mathrm{TS}=200 \mathrm{~ms}$ ). Both pre- and post-contrast datasets were acquired in $\mathrm{n}=10$ subjects. A paired t-test was used to assess the statistical significance between measurements of the SD in a septal ROI. This study was approved by the local Institutional Review Boards of the National Heart, Lung, and Blood Institute and Suburban Hospital, and all subjects gave written informed consent to participate. Post-contrast T1-maps were typically acquired at least $15 \mathrm{~min}$ following administration of $\mathrm{Gd}$ contrast $(0.15 \mathrm{mmol} / \mathrm{kg})$ (Gadavist, Bayer Healthcare).

\section{Results}

Analytic SD validation by Monte-Carlo simulation Using SASHA with protocol NS $+[(0) 1]^{12}$ with uniformly distributed TS, the predicted SD was within $1 \%$ of measured Monte-Carlo SD for $\mathrm{SNR} \geq 30$, and within approx. $2 \%$ of measured SD for SNR $=20$ (Figure 3).

\section{Optimization for known T1}

In the case where $\mathrm{T} 1$ is assumed to be known, brute force optimization found that the sampling strategy of using all measurements with a single fixed value of TS plus a non-saturated anchor image without added recovery beats was a near optimum distribution. For sufficiently large $\mathrm{N}$, low $\mathrm{T} 1$, and low $\mathrm{HR}$, the addition of a single measurement with recovery heart beats to achieve a longer saturation delay was found to reduce the $\mathrm{CV}$. Consider the case of NS +2 measurements as an illustration. The $\mathrm{SD}\left(\mathrm{TS}_{1}, \mathrm{TS}_{2}\right)$ for a protocol with $\mathrm{NS}+2$ measurements $\left(\mathrm{NS}+[(0) 1]^{2}\right)$ is minimum along a line $\mathrm{TS}_{1}=\mathrm{TS}_{2}$ (Figure 4). In this illustration, the saturation delays are not-constrained to be less than the RR interval as they would in a real implementation in order to visualize the surface and various minima. There is a global optimum (arrow A) at $\mathrm{TS}_{1}=\mathrm{TS}_{2}<\mathrm{T} 1$ where TS is relatively short and there is another relative minimum at a point (arrow B) with short $\mathrm{TS}_{1}<\mathrm{T} 1$ and a long $\mathrm{TS}_{2}>>\mathrm{T} 1$ which would necessitate many recovery heart beats to achieve.

Using brute force optimization, a fixed $\mathrm{TS}<\mathrm{T} 1$ was found to be optimum or near optimum over a wide range of parameters. Using $\mathrm{Eq} 5$, the $\mathrm{CV}=\mathrm{SD} / \mathrm{T} 1$ may be evaluated for the proposed sampling strategy of fixed TS. The ratio TS/T1 depends only on the number of measurements $\mathrm{N}$ (Figure $5(\mathrm{a})$ ). For $\mathrm{N}=1$, the optimum saturation delay is approx. $84 \%$ of the $\mathrm{T} 1$ and decreases to $50 \%$ of $\mathrm{T} 1$ at $\mathrm{N}=12$. The normalized coefficient of variation $(\mathrm{SD} / \mathrm{T} 1)^{*} \mathrm{SNR}$ is a decreasing function of $\mathrm{N}$ (Figure $5(\mathrm{~b})$ ). For $\mathrm{N}=12$, the (SD/T1)* $\mathrm{SNR}$ is approx. 1.6; therefore, at $\mathrm{SNR}=40$ and $\mathrm{T} 1=1000$, the $\mathrm{SD}$ is calculated to be $40 \mathrm{~ms}$. Plots for the optimum fixed TS and $\mathrm{SD}$ are shown for $\mathrm{SNR}=40$ and various $\mathrm{N}$ in Figure 5(c) and (d).

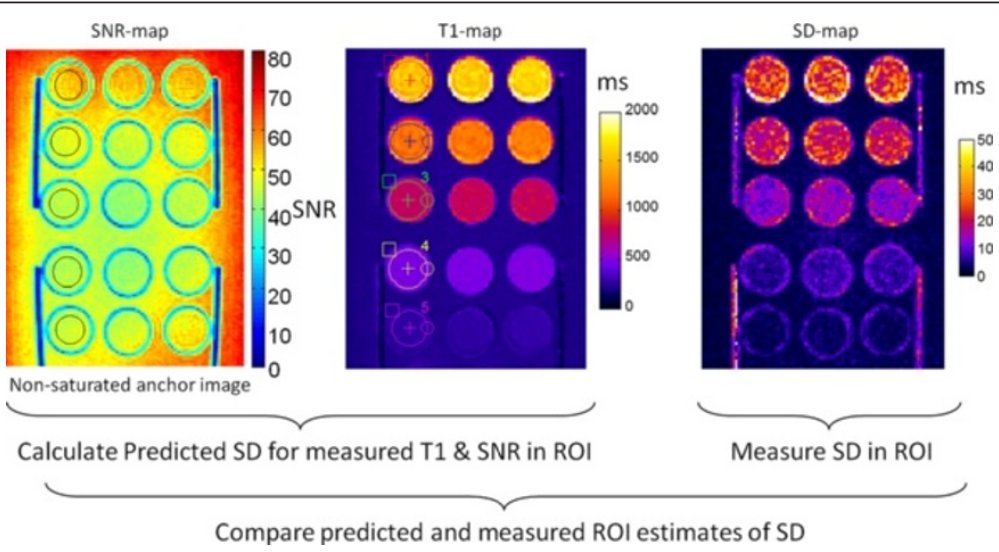

Figure 2 Methodology used for phantom ROI analysis comparing predicted SD based on measured SNR and T1 with measured SD. 


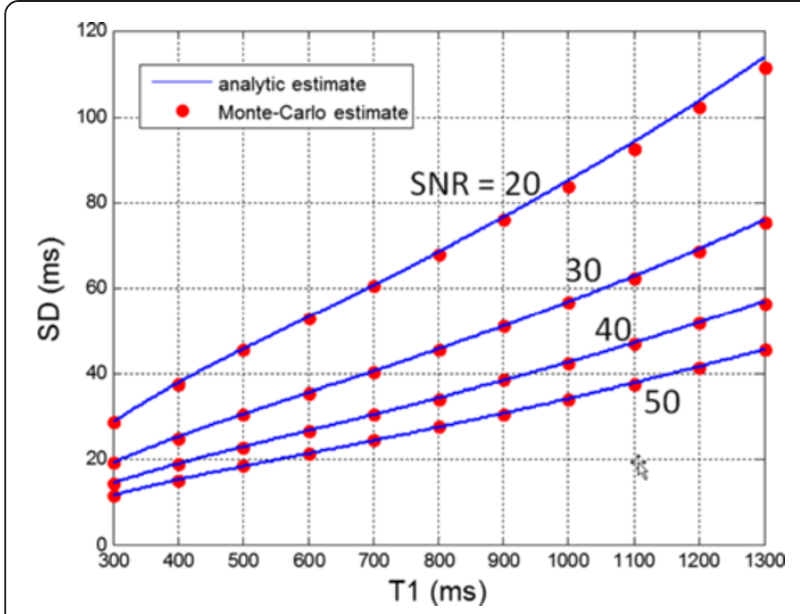

Figure 3 Validation of analytic formulation of SD using Monte-Carlo with $n=65536$ trials demonstrating excellent agreement.

The fixed TS sampling was compared with brute force optimization which permitted recovery heartbeats. Results for calculation of $\mathrm{CV}$ for $\mathrm{N}=9$ and 12 at $\mathrm{SNR}=40$ for various strategies are listed in Table 1, for both $60 \mathrm{bpm}$ and $120 \mathrm{bpm}$ (in parentheses). For $\mathrm{N}=12, \mathrm{~T} 1=$ $1200 \mathrm{~ms}$, the fixed TS strategy is within $2 \%$ of a brute force optimized sampling which has a single measurement with recovery beats, for HRs 60 to $120 \mathrm{bpm}$. The

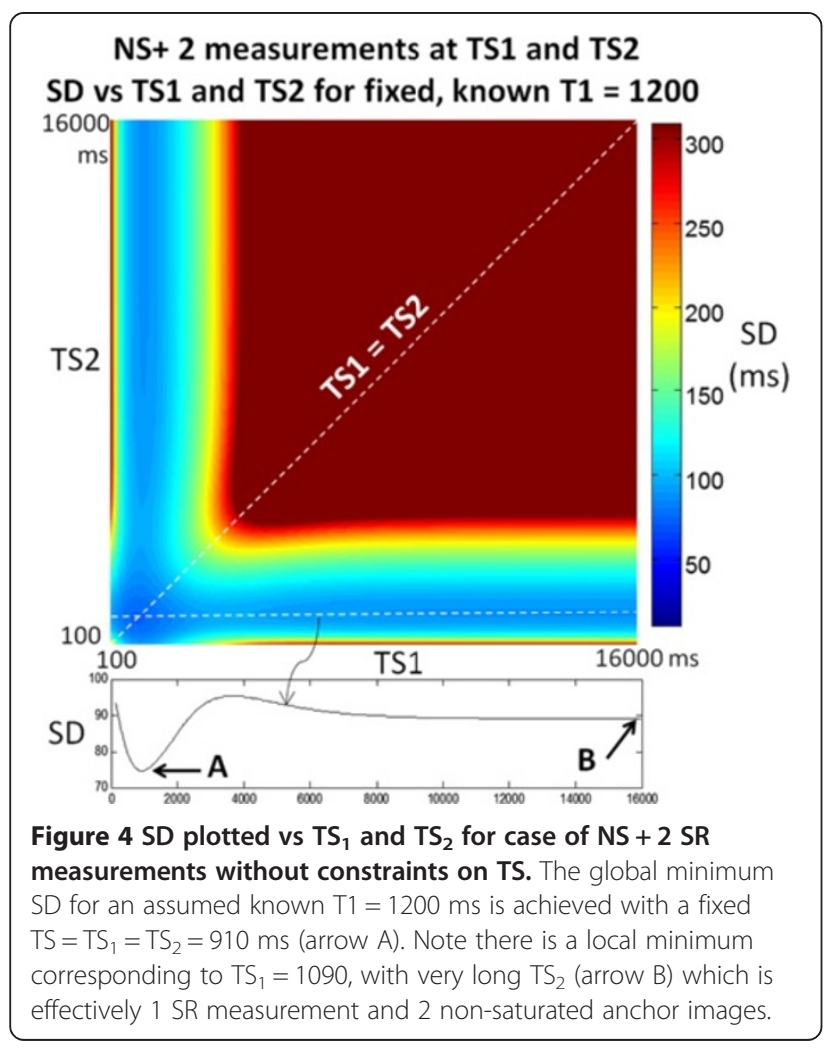

brute force optimization chose 4 recovery heartbeats for 1 measurement, and 7 measurements at fixed TS (i.e., $\left.\mathrm{NS}+[(0) 1]^{7}(4) 1\right)$. For $\mathrm{N}=9, \mathrm{~T} 1=1200 \mathrm{~ms}$, the fixed TS strategy without recovery beats is optimal $\left(\mathrm{NS}+[(0) 1]^{9}\right)$. For $\mathrm{T} 1=500 \mathrm{~ms}$ and $\mathrm{N}=12$, the fixed TS strategy is within $13 \%$ of optimum at $\mathrm{HR}=60 \mathrm{bpm}$ and within $8 \%$ at a $\mathrm{HR}=120 \mathrm{bpm}$. For $\mathrm{T} 1=500 \mathrm{~ms}$ and for $\mathrm{N}=9$, the fixed TS strategy is within $7 \%$ and $1 \%$ at $\mathrm{HRs} 60$ and $120 \mathrm{bpm}$, respectively. For $\mathrm{T} 1=250$ and $\mathrm{N}=12 \mathrm{~ms}$ the fixed TS strategy is within $21 \%$ and $18 \%$ of the brute force optimization including recovery beats at HRs 60 and $120 \mathrm{bpm}$, respectively. Brute force optimization without recovery heart beats, choosing 9 measurements fixed at $150 \mathrm{~ms}$ and 3 measurements fixed at $800 \mathrm{~ms}$ (max), is within $6 \%$ of the best optimization which allows for recovery heart beats. For $\mathrm{T} 1=250 \mathrm{~ms}$ and $\mathrm{N}=9$, the fixed TS strategy is within $12 \%$ and $11 \%$ of the brute force optimization allowing recovery beats at HRs 60 and $120 \mathrm{bpm}$, respectively. Brute force optimization without recovery heart beats, choosing 7 measurements fixed at $150 \mathrm{~ms}$ and 2 measurements fixed at $800 \mathrm{~ms}$ (max), is within $4.5 \%$ of the best optimization which allows for recovery heart beats.

\section{Optimization for unknown T1}

Various sampling strategies were compared for the case of unknown T1 over 3 ranges of T1 corresponding to native $\mathrm{T} 1$ range, post-contrast $\mathrm{T} 1$, and a wide range spanning native and post-contrast. All protocols compared here were 13 heartbeat acquisitions with different sampling. The first set of plots (Figure 6) compare 3 strategies using NS $+[(0) 1]^{12}$ with different distribution of saturation delays: a brute force optimization without recovery heartbeats (blue), uniform distribution from 100 to $800 \mathrm{~ms}$ (red), and fixed TS (green). The fixed TS optimized for each range was 591, 193, and $290 \mathrm{~ms}$ for native, post-contrast, and wide ranges, respectively. The dotted black line is the optimum achievable SD, i.e., using fixed TS for each T1 which serves as a lower bound. Note that the fixed TS achieved approximately the same performance as the brute force optimization over the native contrast and post-contrast ranges, but deviates for the wide range. The saturation delays found by brute force search were (a) native range: NS + [2@550 ms, 10@600 ms], (b) post-contrast range: NS + [11@250 ms, $1 @ 800 \mathrm{~ms}]$ (c) wide range: NS + [4@300 ms, 4@350 ms, $4 @ 800 \mathrm{~ms}]$. Brute force optimization over the native T1 range that included recovery beats chose a strategy of fixed TS sampling without recovery beats. Brute force optimization over the post-contrast $\mathrm{T} 1$ range that included recovery beats chose a strategy with a single recovery beat and was $12 \%$ better than the fixed TS strategy for this T1-range. Brute force optimization 


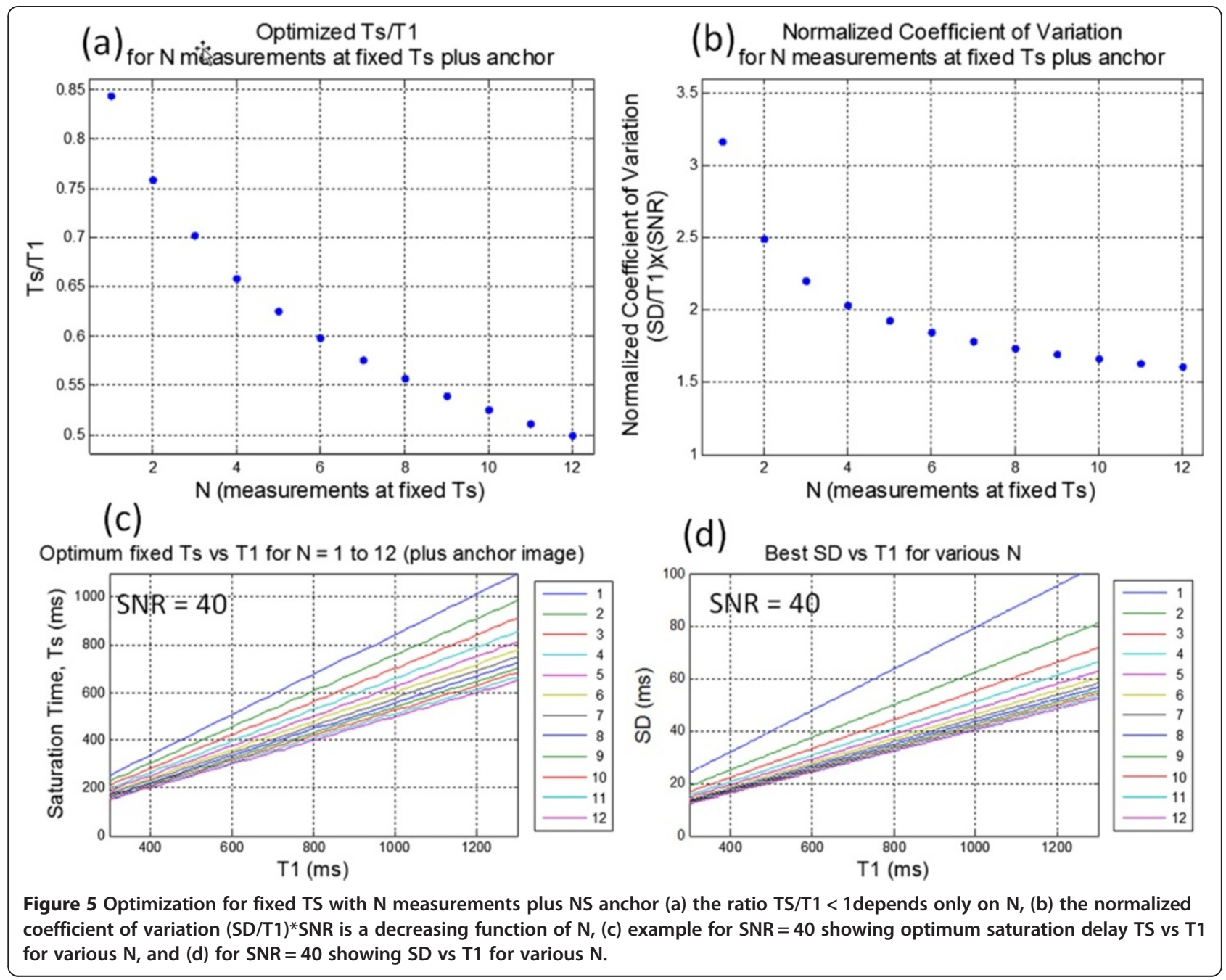

without recovery heart beats was within $8 \%$ of the brute force optimization which allowed recovery, but which increased the saturation delay for a single measurement (11 measurements at $250 \mathrm{~ms}$ and a single measurement at $800 \mathrm{~ms}$ ).

The second set of plots (Figure 7) compare the strategy $\mathrm{NS}+[(0) 1]^{3}(1) 1(2) 1(3) 1$ (magenta) with the NS $+[(0) 1]^{12}$ uniform distribution from 100 to $800 \mathrm{~ms}$ (red), and fixed TS (green) for heart rates (HR) of 60 and $120 \mathrm{bpm}$. The fixed TS optimized for each range are the same as Figure 6 with the exception of the native range at $\mathrm{HR}=120 \mathrm{bpm}$ for which $591 \mathrm{~ms}$ is not achievable; for this case a value of $350 \mathrm{~ms}$ is used. For native contrast and post-contrast ranges the fixed TS strategy has superior performance (lowest coefficient of variation across the range). The NS $+[(0) 1]^{3}(1) 1(2) 1(3) 1$ strategy of recovery beats with uniform TS distribution, is significantly poorer than the fixed TS strategy.

Table 1 Coefficient of variation (CV) for various sampling strategies for both $N=9$ and $N=12$ at $S N R=40$, for $\mathrm{HR}=60 \mathrm{bpm}$ and $120 \mathrm{bpm}$ (values in parentheses)

\begin{tabular}{|c|c|c|c|c|c|c|}
\hline \multicolumn{2}{|l|}{ \# SR measurements } & \multicolumn{3}{|l|}{$\mathrm{N}=9$} & \multicolumn{2}{|l|}{$N=12$} \\
\hline $\mathrm{T1}$ (ms) & 250 & 500 & 1200 & 250 & 500 & 1200 \\
\hline $\mathrm{NS}+[(0) 1]^{\mathrm{N}}$ Uniform & $0.0517(0.0472)$ & $0.0488(0.0442)$ & $0.0461(0.0595)$ & $0.0467(0.0451)$ & $0.0461(0.0415)$ & $0.0429(0.0534)$ \\
\hline$N S+[(0) 1]^{N}$ Fixed TS & $0.0424(0.0424)$ & $0.0424(0.0424)$ & $0.0424(0.0480)$ & $0.0402(0.0402)$ & $0.0402(0.0402)$ & $0.0402(0.0440)$ \\
\hline $\mathrm{NS}+[(0) 1]^{3}(1) 1(2) 1(3) 1$ SMART1Map & N/A & N/A & N/A & $0.0670(0.0512)$ & $0.0564(0.0566)$ & $0.0596(0.0563)$ \\
\hline Brute force optimization without recovery beats & $0.0394(0.0394)$ & $0.0424(0.0424)$ & $0.0424(0.0424)$ & $0.0353(0.0353)$ & $0.0402(0.0402)$ & $0.0402(0.0402)$ \\
\hline Brute force optimization allowing recovery beats & $0.0377(0.0383)$ & $0.0396(0.0419)$ & $0.0424(0.0424)$ & $0.0333(0.0341)$ & $0.0356(0.0374)$ & $0.0394(0.0402)$ \\
\hline
\end{tabular}



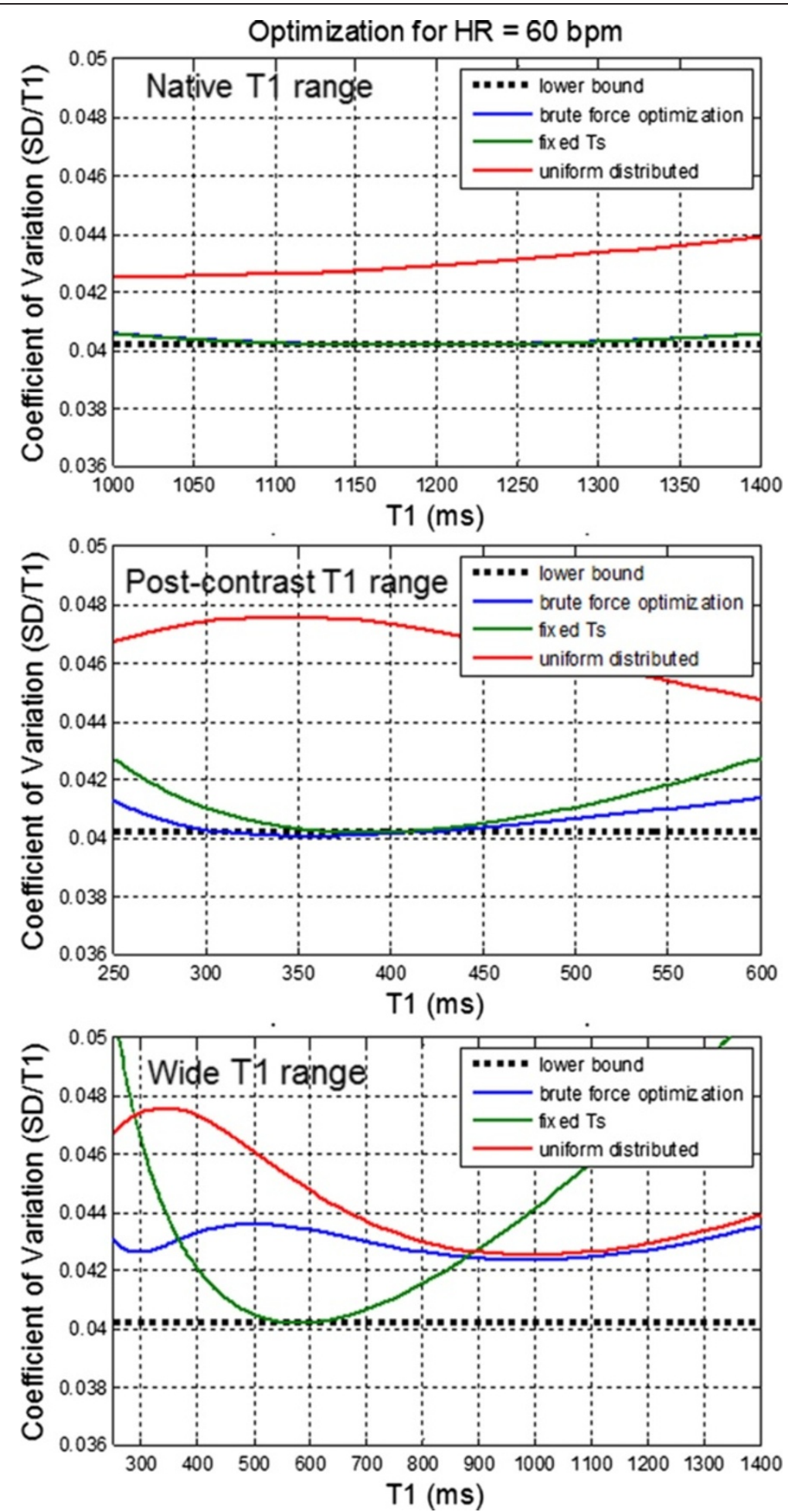

Figure 6 Comparison of sampling strategies using NS $+[(0) 1]^{12}$ with brute force optimization of the sampling scheme, uniform distribution, and fixed saturation delay against the lower bound (dotted) for 3 ranges of $\mathrm{T} 1$ uncertainty.

\section{Phantom measurements}

The sampling strategies compared in Figure 7 were evaluated using a T1-phantom. T1 and SD maps as well as
SNR maps were reconstructed for each protocol and ROI measurements for 5 T1-tubes were compared with respect to measured $\mathrm{SD}$ and theoretical prediction as 


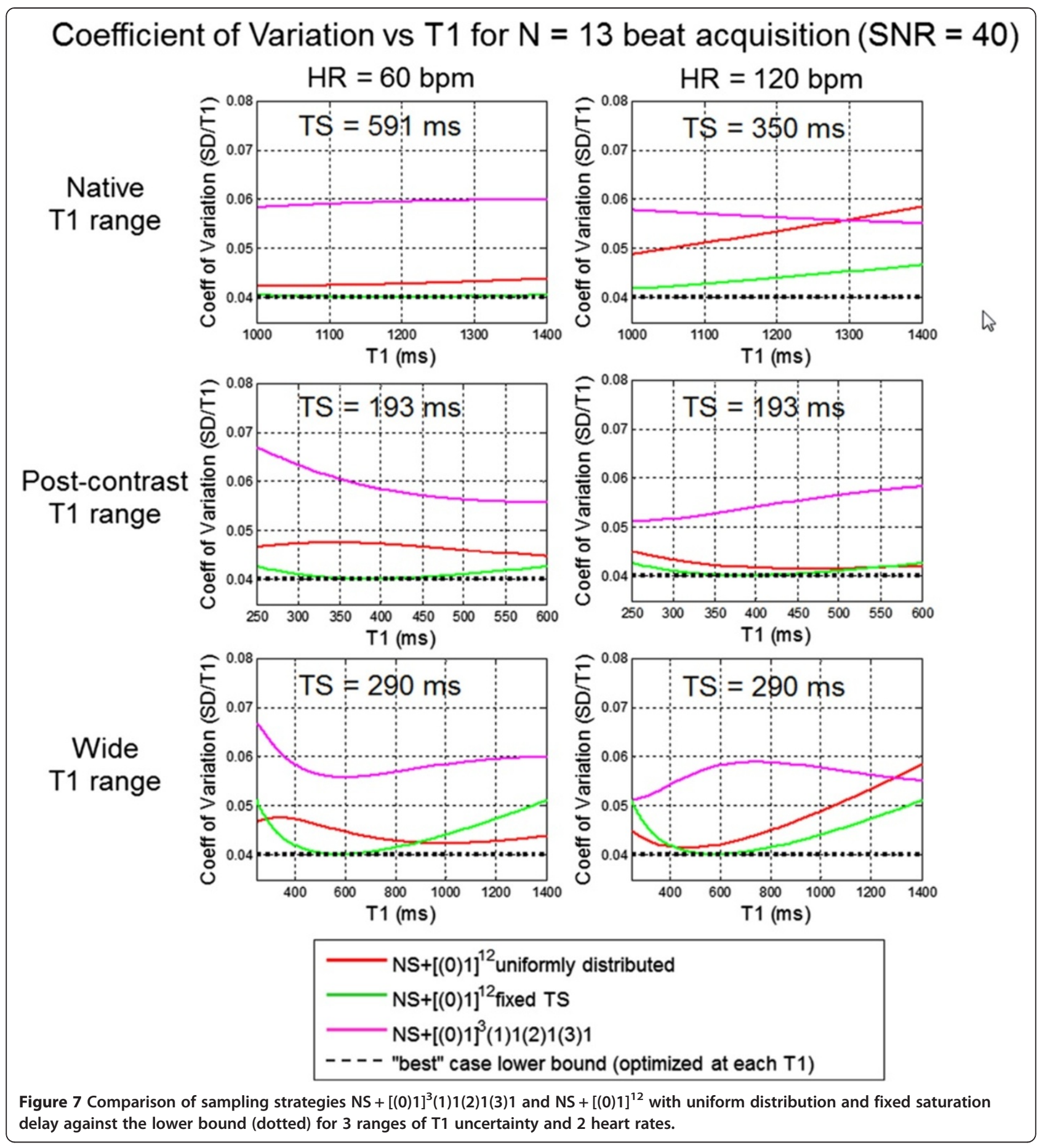

described in Figure 2. Images were acquired at both 60 and $120 \mathrm{bpm}$. The $\mathrm{T} 1$ of the agar gel tubes spanned the range of native myocardial $\mathrm{T} 1$ and for $\mathrm{Gd}$ contrast so fixed saturation delay protocols optimized for the $3 \mathrm{~T} 1$ ranges were used at each heart rate. The TS values were rounded slightly in comparison with the Figure $7 \mathrm{calcu}-$ lations. T1 and SD maps for 60 and $120 \mathrm{bpm}$ are shown in Figures 8 and 9, respectively, and measured values of
$\mathrm{SD}$ in 5 ROIs are graphed in Figure 10. The measured SD agreed well with the predicted SD for a given protocol, T1, and SNR (Figure 11). The SD values for the top rows in Figures 8 and 9 with longer T1 corresponding to native T1 range are smallest for the fixed TS protocol ( $\mathrm{TS}=600 \mathrm{~ms}$ for $\mathrm{HR}=60 \mathrm{bpm}$ and $\mathrm{TS}=350 \mathrm{~ms}$ for $\mathrm{HR}=120 \mathrm{bpm}$ ) as expected from Figure 7. The SD values for the lower rows with shorter T1 corresponding to post 


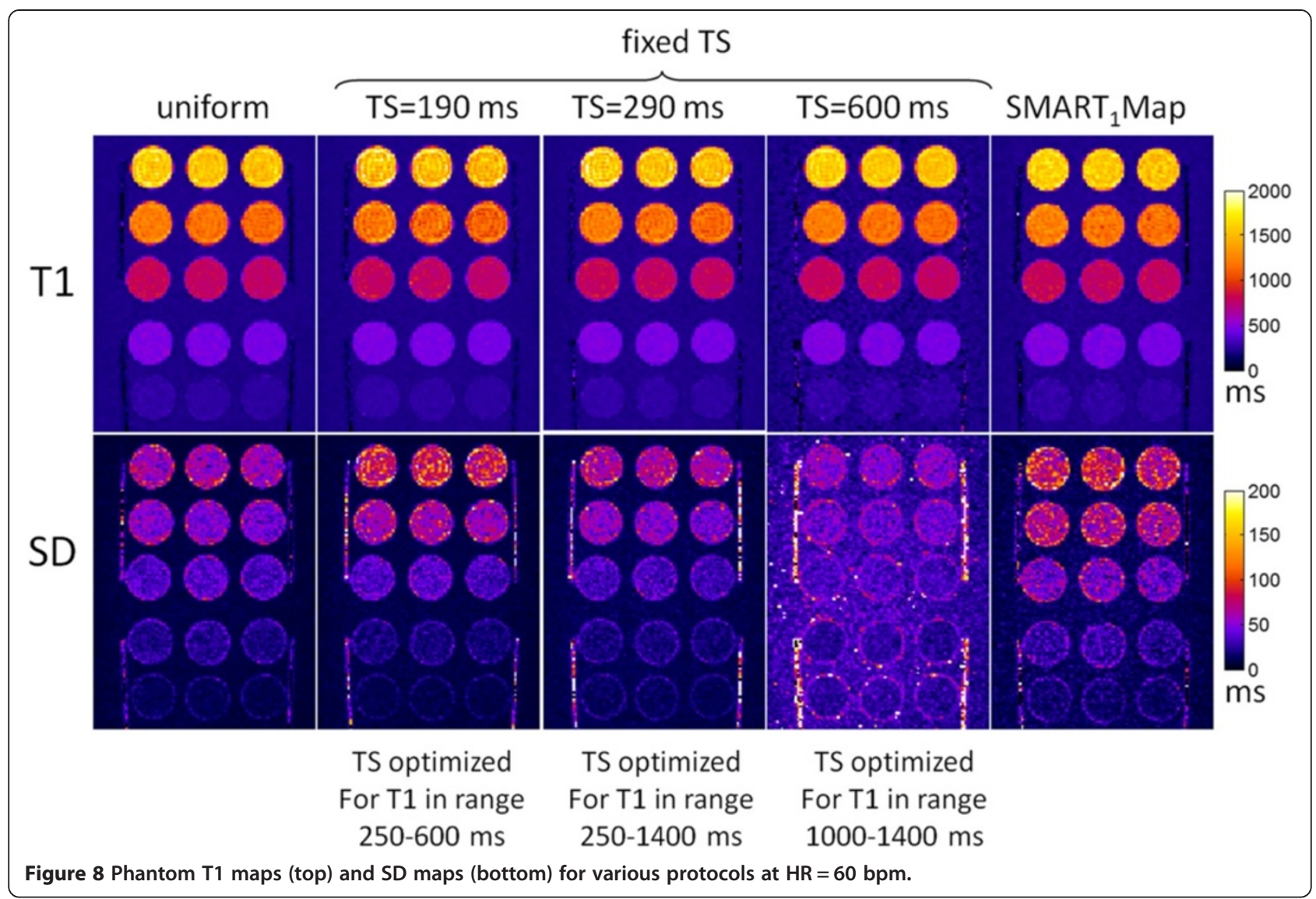

contrast range are smallest for the fixed TS protocol ( $\mathrm{TS}=190 \mathrm{~ms}$ ) at HR=60 and approx. the same as the uniform distribution for $\mathrm{HR}=120 \mathrm{bpm}$, as expected from Figure 7 . The NS $+[(0) 1]^{3}(1) 1(2) 1(3) 1$ protocol performed significantly worse than the optimized fixed TS protocols over the native and post-contrast ranges for which they were respectively optimized.

\section{In-vivo measurements}

T1-maps were acquired with 3 protocols on $n=10$ subjects, both native $\mathrm{T} 1$ (pre-contrast) and post-contrast. Example T1 and SD maps are shown in Figures 12 and 13 , for native and post-contrast examples, respectively. ROI measurements of the SD were made in the septum displayed as box and whisker plots (Figure 14). For precontrast measurements, the SD values for the SMART Map approach are significantly higher than either the original SASHA protocol $(\mathrm{p}=0.012)$ and the modified protocol $(\mathrm{p}=0.0015)$; values for original and modified methods do not reach a statistically significant difference $(\mathrm{p}=0.33)$. For post-contrast measurements, the SD values for the SMART1Map approach are significantly higher than either the original SASHA protocol $(\mathrm{p}<0.001)$ and the modified protocol $(\mathrm{p}<0.001)$; $\mathrm{SD}$ values for the modified protocol are less than the original protocol with statistical significance $(\mathrm{p}<0.03)$ thereby demonstrating that the fixed TS protocols optimized for the specific T1ranges have clearly improved precision.

\section{Discussion}

The analytic formulation for parameter error (Eq 5) adds insight into finding optimal sampling strategies for saturation recovery $\mathrm{T} 1$ mapping. The first term in the denominator represents the uncertainty in an estimate of T1 given that the signal amplitude A is known and would be optimized at TS $=\mathrm{T} 1$ for all $\mathrm{N}$. The 2 parameter fitting jointly estimates $\mathrm{A}$ and $\mathrm{T} 1$, and the second term in the denominator subtracts from the first term leading to an increase in SD and shifts the optimal TS $<$ T1. Sampling with recovery heartbeats decreases the total number of measurements in order to maintain a fixed total duration for acquisition. The SD decreases as $\mathrm{N}$ increases (Figure 5(b)), therefore, recovery heartbeats can cause an increase in SD. However there is a point of diminishing returns where the added measurements do not significantly decrease the SD. At this point, the addition of a measurement with recovery beats can actually decrease the SD. While all of the measurements contribute to the joint estimation of T1 and signal amplitude, the short $\mathrm{TS}<\mathrm{T} 1$ dominate the $\mathrm{T} 1$ estimation, and 


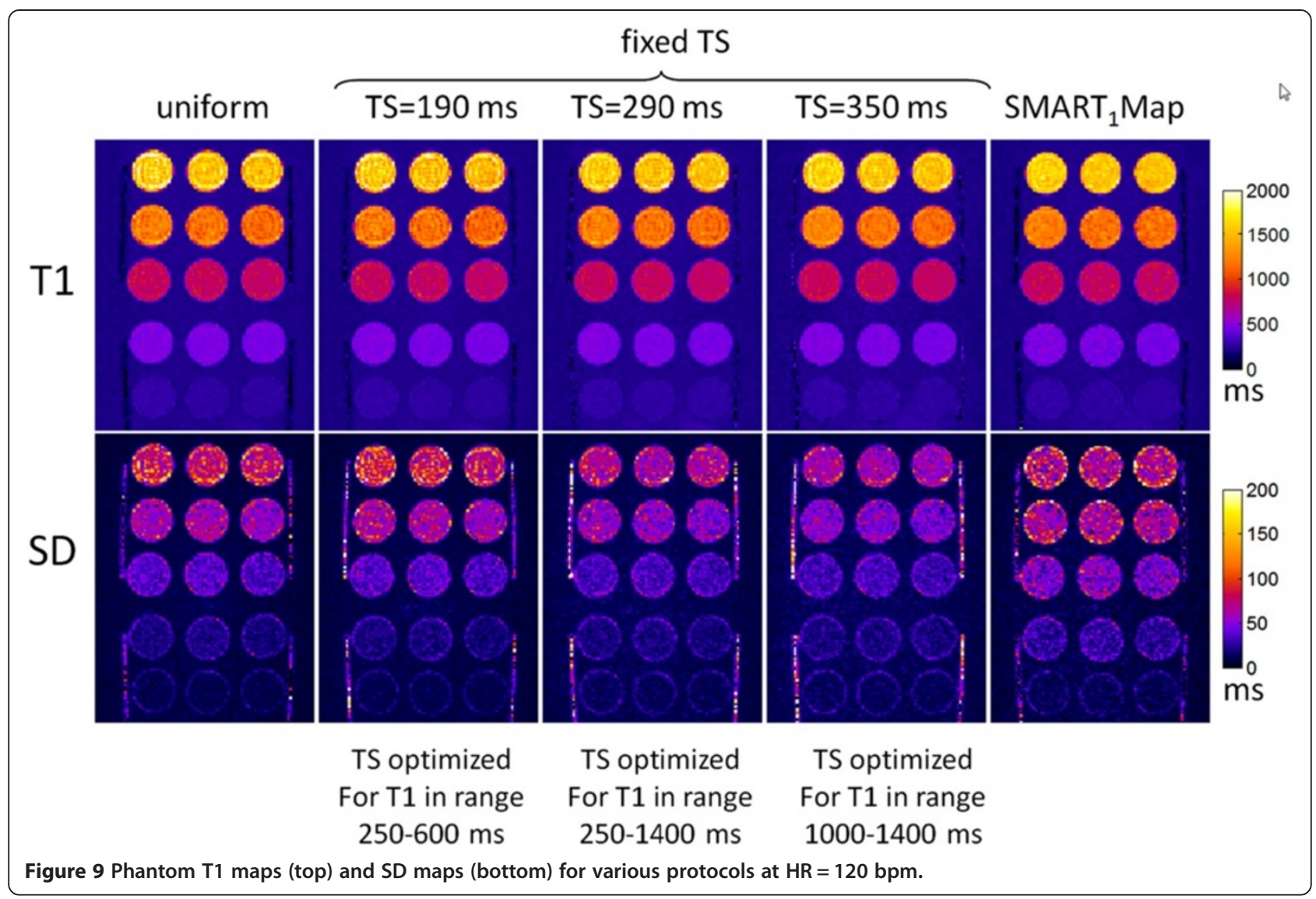

the NS anchor measurement dominates the amplitude estimate. At the point for which there are enough samples, the overall estimation benefits from a longer TS measurement (i.e., acquired at essentially full recovery after several recovery beats) to improve the amplitude estimate. The interplay between the 2 terms in the denominator is complex and depends $\mathrm{N}, \mathrm{T} 1$, and the RR interval whereas the fixed TS sampling strategy is simple to implement.

In this study, we somewhat arbitrarily defined the native and post-contrast $\mathrm{T} 1$ ranges. In the case of native contrast, the range of actual myocardial $\mathrm{T} 1$ values are affected by disease conditions such as edema [16,17], iron deposition
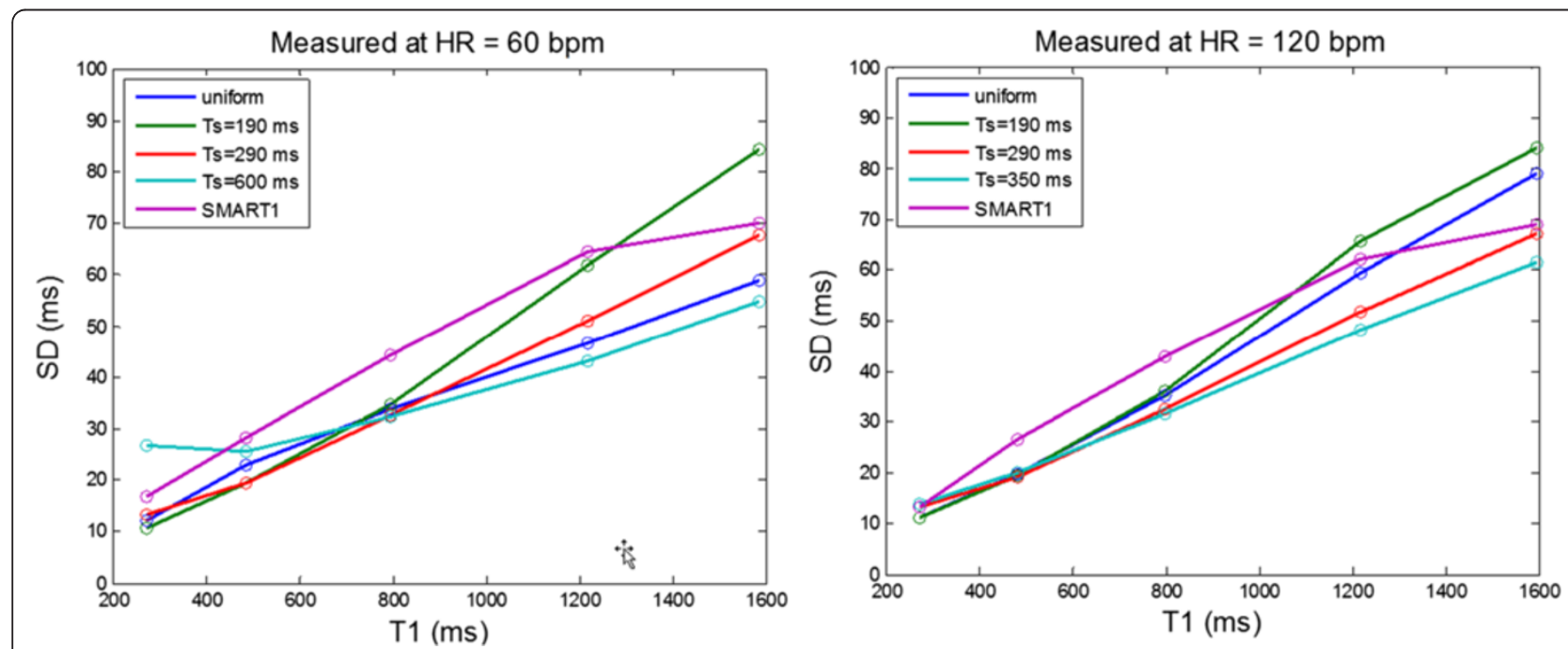

Figure 10 Measured SD for phantom ROls at $\mathrm{HR}=60 \mathrm{bpm}$ (left) and $120 \mathrm{bpm}$ (right). 

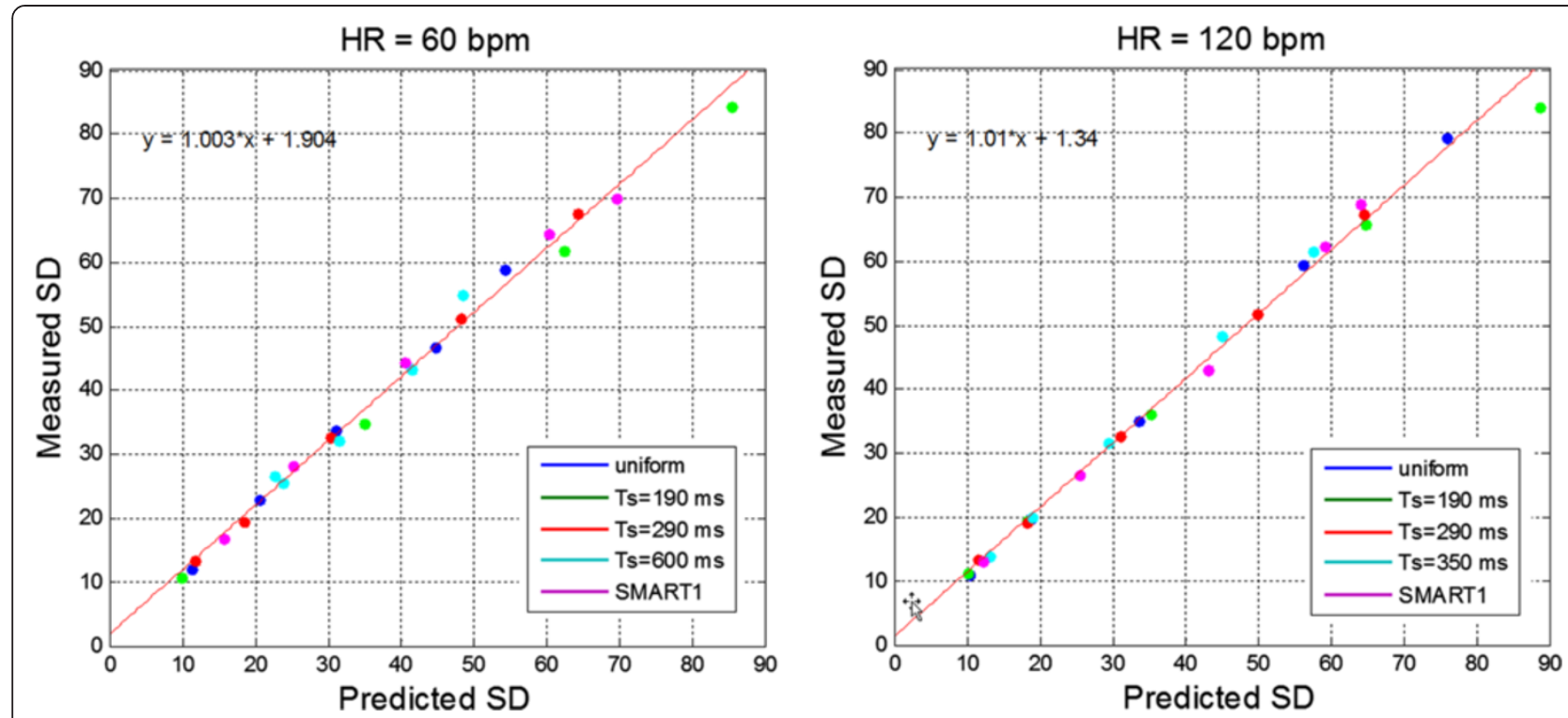

Figure 11 Scatter plots of measured versus predicted SD for phantom ROIs at HR=60 bpm (left) and 120 bpm (right). Measurements plotted for 5T1-tubes with each of 5 protocols (Figure 10), with fit to all of the measured data (red line).

[18], and lipid deposition [19,20]. However, the optimal fixed saturation delay is fairly weakly dependent on the assumed native range $1000-1400 \mathrm{~ms}$. In the case of post-contrast, the assumed $250-600 \mathrm{~ms}$ range is quite large. For a reduced target range for post-contrast T1 of 400-600 ms, the optimum fixed delays would increase to approximately 245 and $265 \mathrm{~ms}$ for NS +12 and NS +9 measurements, respectively. This further strengthens the conclusion that a fixed delay scheme is more optimal than other strategies.

In this study we optimized for the expected myocardial T1 and did not optimize for the blood signal. Although measurement of $\mathrm{T} 1$ in the blood is important for applications such as ECV, the blood signal is measured in a ROI and is generally at high SNR. Precision of the blood estimate is excellent and generally does not limit the ECV precision [11].

In the protocol comparison, the acquisition time was 13 beats for all 3 protocols to allow fair comparison, however shorter breath-holds may be desirable. In this case, the optimum saturation delay will slightly increase as shown in Figure 5. For instance for NS +9 measurements, the optimum saturation delays increase slightly to approx. $210 \mathrm{~ms}$ and $640 \mathrm{~ms}$ for post-contrast and native T1 ranges, respectively. The total acquisition time can be moderately reduced without significantly decreasing $\mathrm{T} 1$

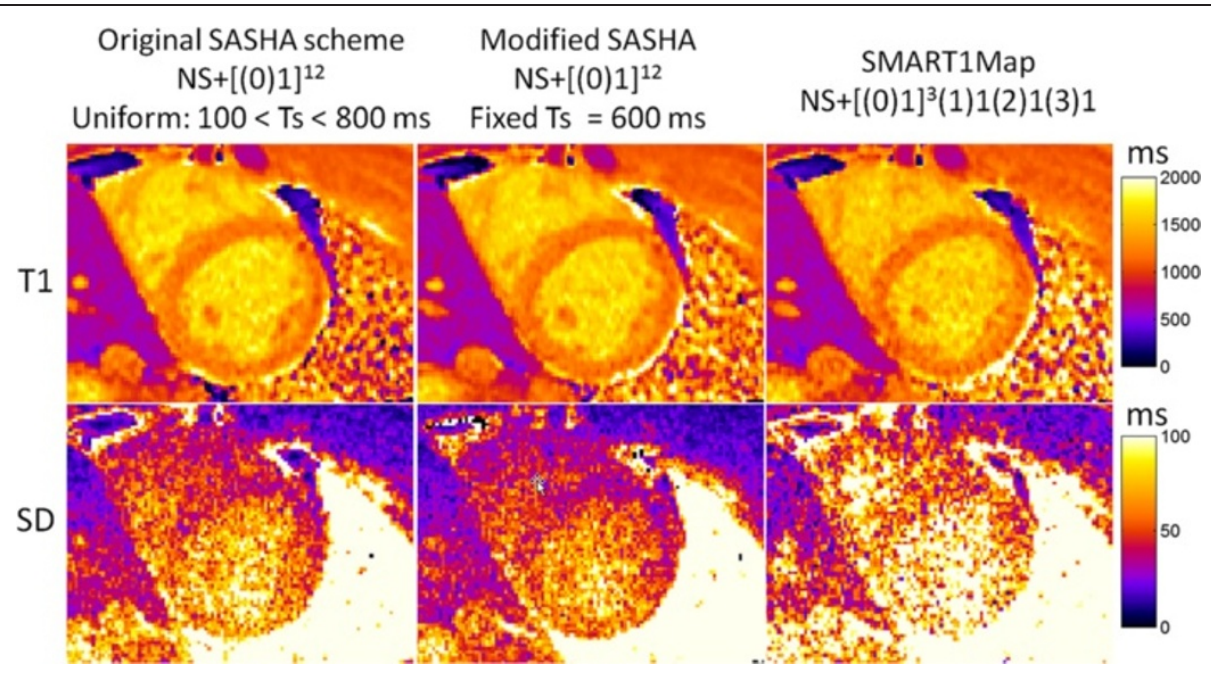

Figure 12 In-vivo example of native T1 maps (top) and SD maps (bottom) using 3 SR-based T1 measurement protocols. 


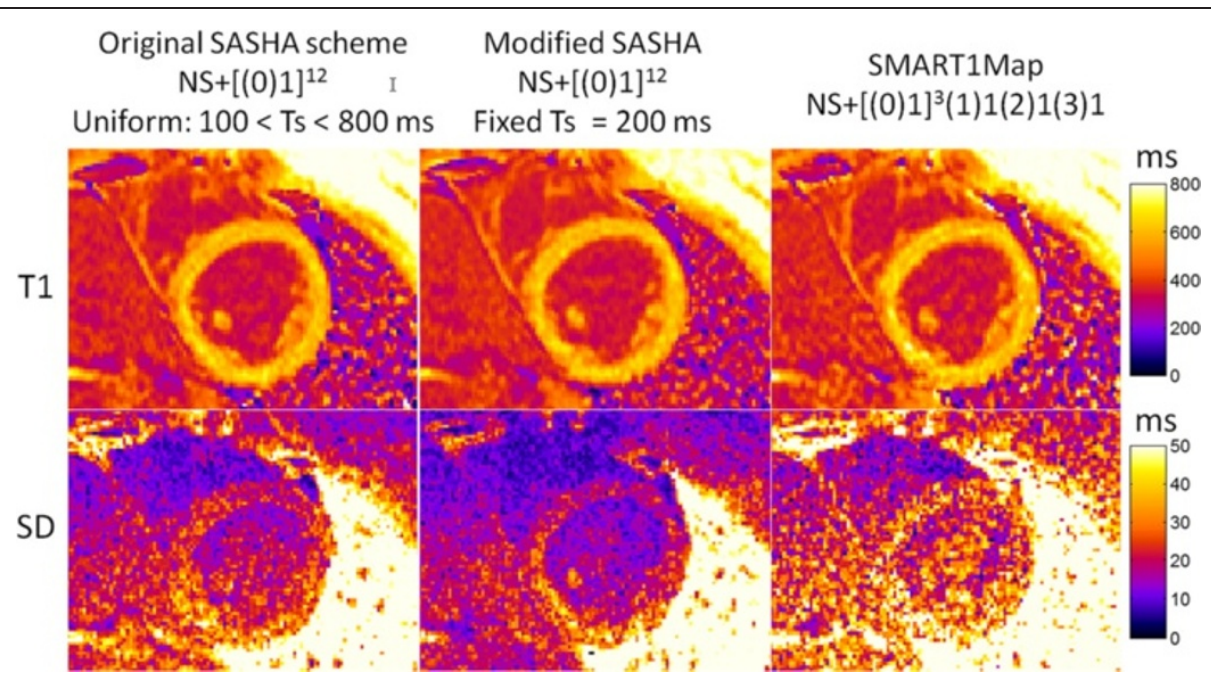

Figure 13 In-vivo example of post-contrast T1 maps (top) and SD maps (bottom) using 3 SR-based T1 measurement protocols.

precision, with only an $8 \%$ increase in SD if the total duration is reduced to 9 beats from 13 (Figure 5(b)).

Three-parameter fitting of SASHA data had substantial increases in T1 variability at high heart rates [3,4]. However, these results show that 2-parameter SASHA maintains excellent precision with heart rates up to $120 \mathrm{bpm}$. The optimal fixed TS for native T1 values is achievable for within a single $R R$ interval for heart rates of less than $80 \mathrm{bpm}$. At higher heart rates where the fixed TS time must be reduced, there is no significant reduction in precision, as demonstrated in simulations and phantom experiments.

This work demonstrates that brute force optimization of sampling including the possible addition of recovery heartbeats for SR T1 mapping does not improve precision as a function of total measurement time for the native T1 range. In the case of the post-contrast $\mathrm{T} 1$ range, there is an improvement in precision when increasing the delay of a couple of measurements. The SMART ${ }_{1}$ Map strategy was previously shown to have less noise than the original SASHA for long T1 values at high HRs [10] when using 3 parameter fitting. In the current study, we have used SASHA 2 parameter fitting which has greatly improved precision compared with 3 parameter [3]. The optimum sampling strategy for 2 parameter fitting does not follow the same behavior as 3 parameter fitting and leads to a different conclusion. The 3-parameter fitting is much more sensitive to the estimation of amplitude terms.

SD maps may be used to compare and optimize protocols. SD maps also serve as a quality metric to assess the individual maps. SD values using the modified protocol have a tighter distribution whereas the SD estimates using the SMART 1 Map method are prone to outliers which are indicative of less reliable curve fitting.

The SASHA VFA with 2-parameter fitting has greatly improved performance over the prior SASHA with fixed

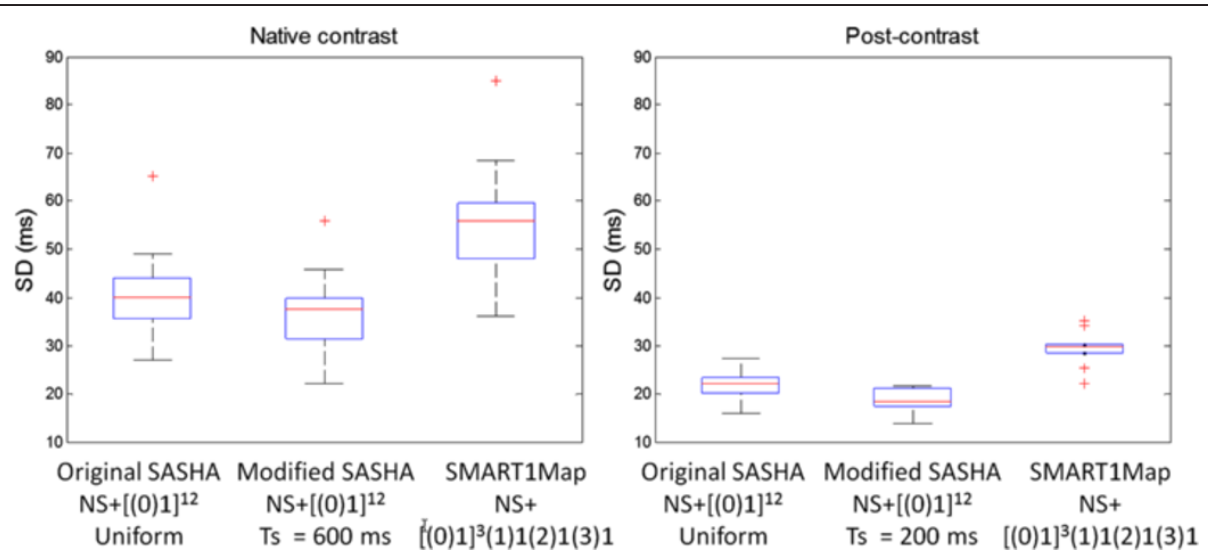

Figure 14 Box and whisker plots of SD values $(n=10)$ in septal ROI comparing the 3 protocols for native contrast (left) and post-contrast (right). Blue box represents interquartile range (IQR) with redline indicating the median. 
excitation flip angle. Benefits of the VFA implementation are (1) a reduction of image artifacts which leads to an improved reproducibility, (2) improved SNR which benefits precision, and (3) reduction of the influence of readout which mitigates the loss in accuracy previously associated with 2-parameter fitting. With these benefits, the SASHA VFA 2-parameter method becomes highly attractive and competitive with inversion recovery protocols such as MOLLI [5]. From an absolute precision standpoint, the MOLLI protocols $5 \mathrm{~s}(3 \mathrm{~s}) 3 \mathrm{~s}$ and $4 \mathrm{~s}(1 \mathrm{~s}) 3 \mathrm{~s}(1 \mathrm{~s}) 2 \mathrm{~s}$ used for native contrast and post-contrast acquisitions [3], respectively, are about $32 \%$ better than the optimized SASHA VFA with the same 11 heartbeat acquisition duration. However, the absolute accuracy of SASHA is superior to the MOLLI approach. More important than absolute accuracy is the reduced dependence of SASHA on variations in off-resonance and flip angle [3]. For native contrast, variations in off-resonance and flip angle can lead to artifactual variation in apparent T1 on the order of $5 \%$ or more when using MOLLI which is greater than the loss precision which is typically less than $2 \%$ on a pixel-wise basis. Respiratory motion correction of inversion recovery data is also more prone to errors than saturation recovery methods due to the large variation in image contrast.

\section{Conclusions}

Sampling strategies for saturation recovery methods for myocardial T1-mapping have been optimized and validated experimentally. Improved precision may be achieved by using fixed saturation delays when considering native myocardium and post-contrast $\mathrm{T} 1$ ranges. The optimized TS was $591 \mathrm{~ms}, 193 \mathrm{~ms}$, and $290 \mathrm{~ms}$ for native, postcontrast, and wide myocardial $\mathrm{T} 1$ ranges, respectively. Pixel-wise estimates of T1 mapping errors have been formulated and validated for SR fitting methods. The ability to quantify the measurement error has potential to determine the statistical significance of subtle abnormalities that arise due to diffuse disease processes involving fibrosis and/or edema and is useful both as a confidence metric for overall quality, and in optimization and comparison of imaging protocols.

\footnotetext{
Abbreviations

MOLLI: Modified look-locker inversion recovery; SASHA: Saturation recovery single-shot acquisition; SMART ${ }_{1}$ Map: Saturation method using adaptive recovery times for cardiac T1 mapping; SR: Saturation recovery; TS: Saturation time; ROI: Region-of-interest; SD: Standard deviation; CV: Coefficient of variation; NS: Non-saturated.
}

\section{Competing interests}

Dr. Spottiswoode is employed by Siemens Medical Solutions. Dr. Arai is a principal investigator on a US government Cooperative Research And Development Agreement (CRADA) with Siemens Medical Solutions (HL-CR-05-004).

\section{Authors' contributions}

PK conceived of the study, contributed to the formulation and evaluation of algorithms, performed processing and analysis, and drafted the manuscript. HX, KC, RBT contributed to the formulation of algorithms. BSS, KC, PK contributed to sequence development. AEA was responsible for human studies. All authors participated in revising the manuscript and read and approved the final manuscript.

\section{Acknowledgments}

The authors acknowledge Christine Mancini for her exceptional support of technical developments.

\section{Funding}

Supported by the National Heart, Lung and Blood Institute, National Institutes of Health by the Division of Intramural Research, NHLBI, NIH, DHHS (HL004607-14CPB).

\section{Author details}

${ }^{1}$ National Heart, Lung, and Blood Institute, National Institutes of Health, DHHS, 10 Center Drive MSC-1061, Bethesda, MD 20892, USA. ${ }^{2}$ Siemens Medical Solutions, Chicago, USA. '3 Department of Biomedical Engineering, University of Alberta, Edmonton, Canada.

Received: 7 May 2014 Accepted: 9 July 2014

Published online: 04 September 2014

\section{References}

1. Moon JC, Messroghli DR, Kellman P, Piechnik SK, Robson MD, Ugander M, Gatehouse PD, Arai AE, Friedrich MG, Neubauer S, Schulz-Menger J, Schelbert EB. Myocardial T1 mapping and extracellular volume quantification: a Society for Cardiovascular Magnetic Resonance (SCMR) and CMR Working Group of the European Society of Cardiology consensus statement. J Cardiovasc Magn Reson. 2013; 15:92.

2. Higgins DM, Moon JC. Review of T1 mapping methods: comparative effectiveness including reproducibility issues. Curr Cardiovasc Imaging Rep. 2014; 7:9252.

3. Kellman P, Hansen MS. T1-mapping in the heart: accuracy and precision. J Cardiovasc Magn Reson. 2014; 16:2.

4. Chow K, Flewitt J a, Green JD, Pagano JJ, Friedrich MG, Thompson RB. Saturation recovery single-shot acquisition (SASHA) for myocardial T1 mapping. Magn Reson Med. 2013; 00:1-14.

5. Messroghli DR, Radjenovic A, Kozerke S, Higgins DM, Sivananthan MU, Ridgway JP. Modified look-locker inversion recovery (MOLLI) for high-resolution T1 mapping of the heart. Magn Reson Med. 2004; 52:141-6.

6. Robson MD, Piechnik SK, Tunnicliffe EM, Neubauer S. T1 measurements in the human myocardium: the effects of magnetization transfer on the SASHA and MOLLI sequences. Magn Reson Med. 2013; 670:664-70.

7. Chow K, Spottiswoode BS, Pagano JJ, Thompson RB. Improved precision in SASHA T1 mapping with a variable flip angle readout. J Cardiovasc Magn Reson. 2014; 16 Suppl 1: M9.

8. Wacker CM, Bock M, Hartlep AW, Beck G, van Kaick G, Ertl G, Bauer WR, Schad LR. Changes in myocardial oxygenation and perfusion under pharmacological stress with dipyridamole: assessment using T*2 and T1 measurements. Magn Reson Med. 1999; 41:686-95.

9. Slavin GS. Breath-held myocardial T1 mapping using multiple single-point saturation recovery. Proc ISMRM. 2012; 20:1244.

10. Stainsby JA, Slavin GS. Comparing the accuracy and precision of SMART1Map, SASHA and MOLLI. J Cardiovasc Magn Reson. 2014; 16 Suppl 1: 11.

11. Kellman P, Arai $A E, X u e H . T 1$ and extracellular volume mapping in the heart: estimation of error maps and the influence of noise on precision. J Cardiovasc Magn Reson. 2013; 15:56.

12. Akcakaya M, Weingartner S, Manning WJ, Nezafat R. Selection of sampling points for saturation recovery based myocardial T1 mapping. J Cardiovasc Magn Reson. 2014; 16 Suppl 1: W32.

13. Akçakaya M, Weingärtner S, Roujol S, Nezafat R. On the selection of sampling points for myocardial T 1 mapping. Magn Reson Med 2014 00:n/a-n/a. doi:10.1002/mrm.25285.

14. Kellman P, McVeigh ER. Image reconstruction in SNR units: a general method for SNR measurement. Magn Reson Med. 2005; 54:1439-47. 
15. Kellman P. Erratum to Kellman P, McVeigh ER. Image reconstruction in SNR units: a general method for SNR measurement. Magn Reson Med. 2005;54:1439-1447. Magn Reson Med. 2007; 58:211-2.

16. Ugander M, Bagi PS, Oki AJ, Chen B, Hsu L-Y, Aletras AH, Shah S, Greiser A, Kellman P, Arai AE. Myocardial edema as detected by pre-contrast T1 and T2 CMR delineates area at risk associated with acute myocardial infarction. JACC Cardiovasc Imaging. 2012; 5:596-603.

17. Dall'armellina E, Piechnik SK, Ferreira VM, Le Si Q, Robson MD, Francis JM, Cuculi F, Kharbanda RK, Banning AP, Choudhury RP, Karamitsos TD, Neubauer S. Cardiovascular magnetic resonance by non contrast T1 mapping allows assessment of severity of injury in acute myocardial infarction. J Cardiovasc Magn Reson. 2012; 14:15.

18. Feng Y, He T, Carpenter J-P, Jabbour A, Alam MH, Gatehouse PD, Greiser A, Messroghli D, Firmin DN, Pennell DJ. In vivo comparison of myocardial T1 with T2 and T2* in thalassaemia major. J Magn Reson Imaging. 2013; 38:588-93.

19. Thompson RB, Chow K, Khan A, Chan A, Shanks M, Paterson I, Oudit GY. $T_{1}$ mapping with cardiovascular MRI is highly sensitive for Fabry disease independent of hypertrophy and sex. Circ Cardiovasc Imaging. 2013; 6:637-45.

20. Sado DM, White SK, Piechnik SK, Banypersad SM, Treibel T, Captur G, Fontana M, Maestrini V, Flett AS, Robson MD, Lachmann RH, Murphy E, Mehta A, Hughes D, Neubauer S, Elliott PM, Moon JC. Identification and assessment of Anderson-Fabry disease by cardiovascular magnetic resonance noncontrast myocardial T1 mapping. Circ Cardiovasc Imaging. 2013; 6:392-8.

doi:10.1186/s12968-014-0055-3

Cite this article as: Kellman et al: Optimized saturation recovery protocols for T1-mapping in the heart: influence of sampling strategies on precision. Journal of Cardiovascular Magnetic Resonance 2014 16:55.

\section{Submit your next manuscript to BioMed Central and take full advantage of:}

- Convenient online submission

- Thorough peer review

- No space constraints or color figure charges

- Immediate publication on acceptance

- Inclusion in PubMed, CAS, Scopus and Google Scholar

- Research which is freely available for redistribution 